\section{CARIES INFILTRATION THERAPY}

Icon from DMG UK is a treatment for incipient caries and carious white spot lesions, without drilling.

Icon is an innovative caries infiltration therapy. Its micro-invasive technology reinforces and stabilises demineralised enamel without the need for drilling or sacrificing healthy tooth structure. Icon's micro-invasive infiltration technology can be used to treat smooth surface and proximal carious lesions up to the first third of dentine. In just one visit Icon can arrest the progression of early enamel lesions and remove carious white spot lesions.

It enables dentists to offer an immediate treatment without unnecessary loss of healthy tooth structure. It prevents lesion progression and increases life expectancy for the tooth. Providing a highly aesthetic solution, carious white spot lesions treated with Icon take on the appearance of the surrounding healthy enamel. Total treatment time is about 15 minutes.

\section{Reader response number 58}

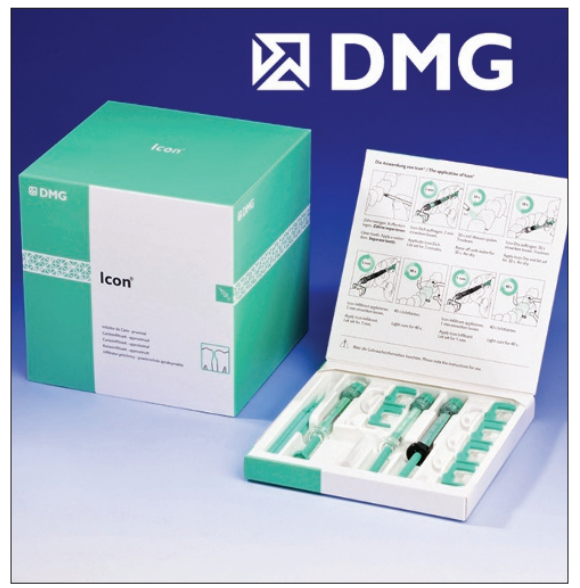

\title{
RAPID STERILISATION PROGRAMME
}

The Sirona DAC Universal combination autoclave cleans, lubricates and sterilises six instruments in 12 minutes. It supports the practice staff by automatically cleaning, lubricating and sterilising handpieces intended for non-critical, semi-critical and critical applications.

The DAC Professional is the ideal complement to Sirona's DAC Universal and Nitraseal systems. The function of the Nitraseal unit is to wrap instruments prior to sterilisation in the DAC Professional.

The DAC Professional can accommodate up to six trays and can be preheated, which significantly reduces cycle times. The rapid sterilisation programme can be completed in as little as ten minutes. It is a Class B steriliser in accordance with EN 13060, and fulfills the stringent requirements laid down for large-capacity sterilisers deployed in hospitals.

It is designed to process difficult materials (for example, $\mathrm{A}+\mathrm{B}$ category hollow objects, surgical cassettes and textiles). During the sterilisation cycle a microprocessor continuously monitors the parameters time, pressure and temperature. This ensures the highest levels of quality and sterilisation security.

Easy to install, all that is required is a mains power supply. The fresh water and waste water tanks are integrated into the steriliser unit. The fresh water level is continuously monitored in order to eliminate errors and prevent damage to the instruments and/or steriliser.

In accordance with the hygiene regulations each sterilisation cycle can be documented by means of a printer or PC software. In this way the clinic or dental practice can safeguard itself against subsequent recourse claims.

Reader response number 59

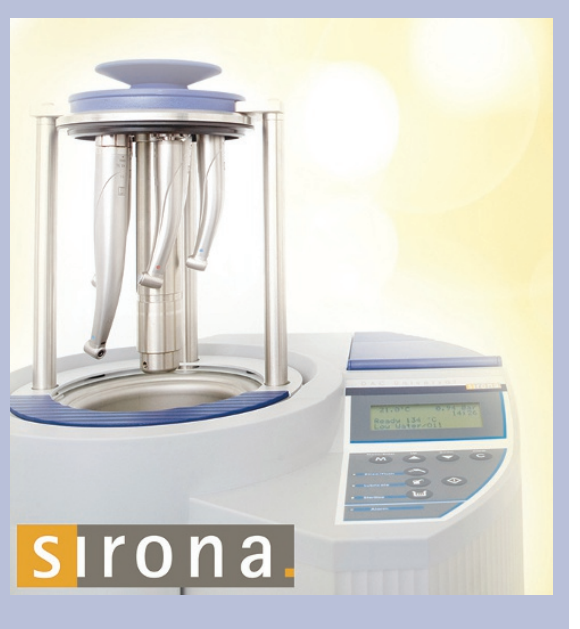

